\title{
Optimal fractal dimension on grain structure robot laser-hardened tool steel
}

\author{
Babic, M. ${ }^{\mathrm{a},}{ }^{*}$, Balic, J. ${ }^{\mathrm{b}}$, Kokol, P. ${ }^{\mathrm{c}}$ \\ ${ }^{a}$ EMO-Orodjarna d.o.o., Celje, Slovenia \\ ${ }^{b}$ Faculty of Mechanical Engineering, University of Maribor, Slovenia \\ ${ }^{c}$ Faculty of Electrical Engineering and Computer Science, University of Maribor, Slovenia
}

\section{A B S T R A C T}

In order to optimize the structure and properties of tool steel, it is necessary to take into account the effect of the self-organization of a dissipative structure with fractal properties at load. Fractal material science researches the relationship between the parameters of fractal structures and the dissipative properties of tool steel. This paper describes the application of fractal dimension in robot laser-hardening specimens. By using fractal dimensions, changes in the structure can be determined because the fractal dimension is a present indicator of the complexities of the sample forms. We hardened tool steel at different speeds and different temperatures. By researching the fractal dimensions of the microstructures of the hardened specimens we could better understand the effects of the parameters of robot cells on the material. We show the experimental results and an analysis of those fractal patterns that occur during robot laser hardening with the different parameters of temperature and speed. Finally, we present the relationship between the fractal dimensions and the parameters of temperature and speed of robot laser hardening. The hardening of various metal alloys showed that when melting occurs, fractal geometry can be used to calculate the fractal dimension. The dependence of the fractal dimension on the hardness was ascertained. This finding is important when we realize that certain alloys mix poorly because they have different melting temperatures but such alloys have a much higher hardness and better technical characteristics.
\end{abstract}

\section{ARTICLE INFO}

Keywords:

Fractal dimension

Robot

Laser

Hardening

*Corresponding author: babicster@gmail.com

(Babic, M.)

Article history:

Received 31 October 2013

Revised 13 May 2014

Accepted 7 July 2014 


\section{References}

[1] Babič, M., Panjan, P., Kokol, P., Zorman, M., Belič, I., Verbovšek, T. (2013). Using fractal dimensions for determination of porosity of robot laser-hardened specimens, International Journal of Computer Science Issues, Vol. 10, No. 2, 184-190.

[2] Mandelbrot, B.B. (1982). The fractal geometry of nature, W.H. Freeman and Company, New York.

[3] Babič, M., Milfelner, M., Belič, I., Kokol, P. (2013). Problems associated with a robot laser cell used for hardening, Materials and Technology, Vol. 47, No. 1, 37-41.

[4] Vollertsen, F., Partes, K., Meijer, J. (2005). State of the art of laser hardening and cladding, In: Proceedings of the $3^{\text {rd }}$ International WLT-Conference on Lasers in Manufacturing, Munich, Germany, 281-305.

[5] Pashby, I.R., Barnes, S., Bryden, B.G. (2003). Surface hardening of steel using a high power diode laser, Journal of Materials Processing Technology, Vol. 139, No. 1-3, 585-588, doi: 10.1016/S0924-0136(03)00509-0.

[6] Tesar, J., Vacikova, P., Soukup, O., Houdkova, S. (2012). Infrared camera analysis of laser hardening, Advances in Optical Technologies, Vol. 2012, 1-6, doi: 10.1155/2012/593893.

[7] El-Batahgy, A.-M., Ramadan, R.A., Moussa, A.-R. (2013). Laser surface hardening of tool steels - experimental and numerical analysis, Journal of Surface Engineered Materials and Advanced Technology, Vol. 3, No. 2, 146-153, doi: 10.4236/isemat.2013.32019.

[8] Lima, M.S.F., Vasconcelos, G., Riva, R. (2012). Laser surface engineering as a tool for more efficient satellite components, Journal of Aerospace Engineering, Sciences and Applications, Vol. 4, No. 4, 105-111.

[9] Feder, J. (1988). Fractals, Springer, New York.

[10] Addison, P.S. (1997). Fractals and chaos: an illustrated course, Institute of Physics Publishing, London.

[11] Xie, H.P., Liu, J.F., Ju, Y., Li, J., Xie, L.Z. (2011). Fractal property of spatial distribution of acoustic emissions during the failure process of bedded rock salt, International Journal of Rock Mechanics and Mining Sciences, Vol. 48, No. 8, 1344-1351, doi: 10.1016/j.ijrmms.2011.09.014.

[12] Li, J., Saharan, A., Koric, S., Ostoja-Starzewski, M. (2012). Elastic-plastic transition in three-dimensional random materials: massively parallel simulations, fractal morphogenesis and scaling functions, Philosophical Magazine, Vol. 92, No. 22, 2733-2758, doi: 10.1080/14786435.2012.674223.

[13] Li, J., Ostoja-Starzewski, M. (2014). Fractal shear bands at elastic-plastic transitions in random Mohr-Coulomb materials, Journal of Engineering Mechanics, Vol. 140, No. 9, 04014072 (just released), doi: 10.1061/(ASCE)EM. 1943-7889.0000750.

[14] Chaudhari, A., Sanders Yan, C.-C., Lee, S.-L. (2004). Multifractal analysis of growing surfaces, Applied Surface Science, Vol. 238, No. 1-4, 513-517, doi: 10.1016/j.apsusc.2004.05.247.

[15] Yu, H.-S., Sun, X., Luo, S.-F., Wang, Y.-R., Wu, Z.-Q. (2002). Multifractal spectra of atomic force microscope images of amorphous electroless Ni-Cu-P alloy, Applied Surface Science, Vol. 191, No. 1-4, 123-127, doi: 10.1016/ S01694332(02)00170-8.

[16] Chen, Z.W., Lai, J.K.L., Shek, C.H. (2005). Multifractal spectra of scanning electron microscope images of $\mathrm{SnO}_{2}$ thin films prepared by pulsed laser deposition, Physics Letters A, Vol. 345, No. 1-3, 218-223, doi: 10.1016/j.physleta. 2005.05.104.

[17] Soković, M., Mikuła, J., Dobrzański, L.A., Kopač, J., Koseč, L., Panjan, P., Madejski, J., Piech, A. (2005). Cutting properties of the $\mathrm{Al}_{2} \mathrm{O}_{3}+\mathrm{SiC}_{(\mathrm{w})}$ based tool ceramic reinforced with the PVD and CVD wear resistant coatings, Journal of Materials Processing Technology, Vol. 164-165, 924-929, doi: 10.1016/i.jmatprotec.2005.02.071. 


\title{
APEM
}

\section{Optimalna fraktalna dimenzija mikrostrukture orodnega jekla, utrjenim z robotskim laserskim kaljenjem}

\author{
Babic, M. ${ }^{\mathrm{a},{ }^{*}}$, Balic, J. ${ }^{\mathrm{b}}$, Kokol, P. ${ }^{\mathrm{c}}$ \\ ${ }^{\mathrm{a}}$ EMO-Orodjarna d.o.o., Celje, Slovenia \\ ${ }^{b}$ Faculty of Mechanical Engineering, University of Maribor, Slovenia \\ ${ }^{c}$ Faculty of Electrical Engineering and Computer Science, University of Maribor, Slovenia
}

\section{POVZETEK}

Da bi lahko optimirali strukturo in lastnosti orodnega jekla, moramo upoštevati vpliv samoorganizacije disipativnih struktur $\mathrm{z}$ lastnostmi fraktalov. Fraktalna znanost o materialih raziskuje odnos med parametri fraktalne strukture in disipativnimi lastnostmi orodnega jekla. Članek opisuje uporabo fraktalne dimenzije pri laserskem kaljenju vzorcev s pomočjo robota. Z uporabo fraktalnih dimenzij se lahko določijo spremembe v strukturi, ker je fraktalna dimenzija pokazatelj kompleksnosti oblike vzorcev. Orodno jeklo smo kalili z različnimi hitrostmi pri različnih temperaturah. S študijem fraktalne dimenzije mikrostrukture kaljenih vzorcev, se da bolje razumeti vpliv procesnih parametrov pri kaljenju na proučevan material. V članku prikazujemo eksperimentalne rezultate in analizo fraktalnih vzorcev, ki se pojavijo med robotskim laserskim kaljenjem, izvedenim pri različnih hitrostih in temperaturah. Na koncu predstavljamo še povezavo med fraktalnimi dimenzijami in temperaturami ter hitrostjo kaljenja. Kaljenje različnih kovinskih zlitin je pokazalo, da lahko tedaj, ko se pojavi taljenje, fraktalno geometrijo uporabimo za izračun fraktalne dimenzije. Ugotovljen je bil tudi vpliv fraktalne dimenzije na trdoto. Ta ugotovitev je pomembna, saj vemo, da se nekatere zlitine medsebojno slabo mešajo, ker imajo različne temperature tališč, vendar pa imajo višjo trdoto in boljše tehnične lastnosti.

\section{PODATKI O ČLANKU}

Ključne besede:

Fraktalna dimenzija

Robot

Laser

Kaljenje

*Kontaktna oseba:

babicster@gmail.com

(Babic, M.)

Zgodovina članka:

Prejet 31. oktobra 2013

Popravljen 13. maja 2014

Sprejet 7. julija 2014 